\title{
Primary central nervous system
} lymphoma with intramedullary spinal cord involvement mimicking inflammatory demyelinating disease

Hyunsoo Kim, MD'; Tai-Seung Nam, MD, PhD'; Michael Levy, MD, PhD²; Kyung-Hwa Lee, MD, $\mathrm{PhD}^{3}$; Jahae Kim, MD, $\mathrm{PhD}^{4}$; Seung-Jin Lee, $\mathrm{MD}^{5}$

'Department of Neurology, Chonnam National University Hospital, Chonnam National University Medical School, Gwangju, Republic of Korea

${ }^{2}$ Department of Neurology, Massachusetts General Hospital, Harvard Medical School, Boston, MA, USA

${ }^{3}$ Department of Pathology, Chonnam National University Medical School, Gwangju, Republic of Korea

${ }^{4}$ Department of Nuclear Medicine, Chonnam National University Hospital, Chonnam National University Medical School, Gwangju, Republic of Korea

${ }^{5}$ Department of Radiology, Chonnam National University Hospital, Chonnam National University Medical School, Gwangju, Republic of Korea

\section{Journal of Neurocritical Care \\ CASE REPORT \\ Received: May 14, 2019 \\ Revised: June 14, 2019 \\ Accepted: June 14, 2019 \\ Corresponding Authors: \\ Tai-Seung Nam, MD, PhD \\ Department of Neurology, Chonnam National University Hospital, Chonnam National University Medical School, 42 Jebong-ro, Dong-gu, Gwangju 61469, Republic of Korea \\ Tel: +82-62-220-6171 \\ Fax: +82-62-236-0839 \\ E-mail: nts0022@hanmail.net \\ Michael Levy, MD, PhD \\ Department of Neurology, Massachusetts General Hospital, Harvard Medical School, 114 16th Street, 3rd Floor, Charlestown, MA 02129, USA \\ Tel: +1-443-287-4612 \\ Fax: +1-888-523-4168 \\ E-mail:mlevy11@mgh.harvard.edu}

Background: Spinal cord involvement of primary central nervous system lymphoma (PCNSL) is rare in a young immunocompetent patient and can be misdiagnosed as an inflammatory demyelinating disease (IDD) of the central nervous system.

Case Report: We report a case of PCNSL mimicking IDD in a previously healthy 46-year-old man with weakness in both hands for 1 week. Magnetic resonance imaging (MRI) of the cervical spinal cord revealed contrast-enhancing intraparenchymal and leptomeningeal lesions in the cervical spinal cord and medulla oblongata. Cerebrospinal fluid analysis revealed pleocytosis $\left(37 / \mathrm{mm}^{3}\right)$. The patient's symptoms and lesions improved with corticosteroid treatment. However, he developed semicomatose mentality 5 months later. Brain $\mathrm{MRI}$, ventricular biopsy, and ${ }^{18} \mathrm{~F}$-flurodeoxyglucose positron emission tomography/computed tomography confirmed PCNSL. The patient deceased 3 months later, despite high-dose methotrexate chemotherapy.

Conclusion: Persistent gadolinium-enhancing MRI lesions along the ventricular regions and spinal leptomeninges could differentiate PCNSL involving the spinal cord from IDD in the early stages of the disease.

Keywords: Primary central nervous system lymphoma; Neuromyelitis optica; Multiple sclerosis; Spinal cord; Magnetic resonance imaging

\section{INTRODUCTION}

Primary central nervous system lymphoma (PCNSL) is an un- common form of extranodal non-Hodgkin lymphoma in the central nervous system (CNS), including the brain, eyes, and cerebrospinal fluid (CSF), with no evidence of systemic spread at

(C) 2019 The Korean Neurocritical Care Society

This is an Open Access article distributed under the terms of the Creative Commons Attribution Non-Commercial License (http://creativecommons.org/licenses/by-nc/4.0/) which permits unrestricted noncommercial use, distribution, and reproduction in any medium, provided the original work is properly cited. 
the time of diagnosis [1]. PCNSL develops in immunocompromised patients and is relatively rare in immunocompetent people [2]. PCNSL mainly develops in the elderly (aged 60 years and above) [3]. In addition, involvement of the intramedullary spinal cord in PCNSL is uncommon and accounts for less than $1 \%$ of PCNSL cases [4-6]. Therefore, PCNSL involving the intramedullary spinal cord in a younger immunocompetent patient can be misdiagnosed as an inflammatory demyelinating disease (IDD) of the CNS, such as multiple sclerosis (MS) or neuromyelitis optica spectrum disorder (NMOSD) [7,8]. Early diagnosis and treatment are important, as two-thirds of the immunocompetent PCNSL patients achieve complete response after treatment with methotrexate [2].

Here, we report a young immunocompetent PCNSL patient with intramedullary spinal cord involvement misdiagnosed with IDD in the early stage of the disease. We discuss the clinical and radiologic features differentiating PCNSL from IDD of the CNS.

\section{CASE REPORT}

A 46-year-old man with mild hypertension presented with asymmetric motor weakness (Medical Research Council scale scores: $4-/ 5$ and $4+/ 5$ in the right and left sides, respectively) in both hands for 1 week. Noncontrast magnetic resonance imaging (MRI) of the brain and cervical spinal cord showed faint T2-hyperintensity in the medulla oblongata and cervical spinal cord (Fig. 1), which were overlooked. Three weeks after onset of symptoms, the patient visited our neurology clinic for the etiological diagnosis. Gadolinium (Gd)-enhanced cervical spinal cord MRI showed prominent lesions in the intramedullary cervical spinal cord and medulla oblongata and contrast enhancement along the ventricular region and spinal leptomeninges (Fig. 2). CSF examination revealed lymphocyte-predominant pleocytosis $\left(37 / \mathrm{mm}^{3}\right)$, with an elevated protein level $(132 \mathrm{mg} / \mathrm{dL})$, but CSF cytomorphologic examination was negative for malignancy, and other neuroimmunological tests including antibody to aquaporin 4 (AQP4), were negative. In addition, serologic tests including the thyroid function test, tumor marker tests, viral tests (human immunodeficiency virus, human T-lymphotropic virus type 1, hepatitis C, herpes simplex virus, cytomegalovirus), CSF-Venereal Disease Research Laboratory test, fluorescent treponemal antibody absorption test, and tests for parasitic infection, were negative. The patient was treated with intravenous methylprednisolone for 5 days, followed by an oral taper of prednisone for 3 months under the presumptive diagnosis of IDD. At the end of the corticosteroid treatment course, the patient showed notable
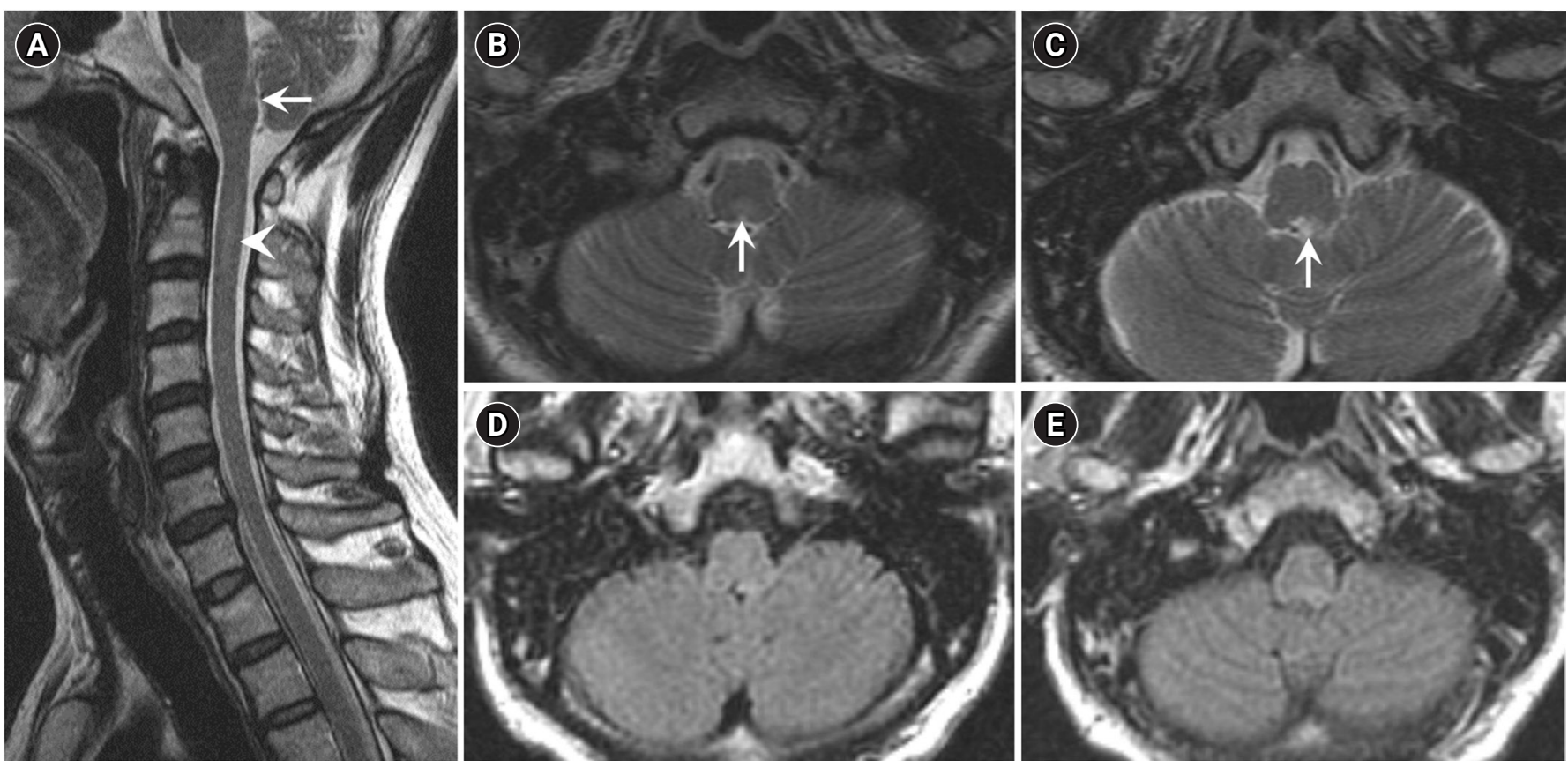

Fig. 1. Initial magnetic resonance imaging (MRI) of the brain and spinal cord 1 week after onset of symptoms. (A) Sagittal and $(B, C)$ axial T2-weighted images show faint hyperintense multifocal lesions in the medulla oblongata (arrows) and cervical spinal cord (arrowhead). (D, E) High signal intensity in the medulla oblongata on T2-weighted images are not clearly detected on fluid-attenuated inversion recovery. No supratentorial or periventricular white matter lesion was seen on brain MRI (images not shown). 
improvement in neurological function. A follow-up cervical MRI revealed significant decrease in parenchymal lesions, except for persistent leptomeningeal enhancement (Fig. 3). Repeat CSF cytomorphologic examination to exclude hematologic malignancy was negative. Five months after completion of the corticosteroid treatment, the patient presented with acute deterioration in mental status. Computed tomography (CT) of the brain revealed a subependymal high-attenuation lesion in all ventricles, with obstructive hydrocephalus and periventricular edema (Fig. 4), and the patient underwent emergency ventriculoperitoneal shunting. Brain MRI showed lesions in the hypothalamus and bilateral periventricular white matter, with subependymal contrast enhancement (Fig. 5). Neuronavigation-guided stereotactic ventricular biopsy confirmed diffuse large B-cell lymphoma (DLBCL), which was positive for B-cell markers, including CD20 and CD79a, but negative for the representative T-cell marker CD3 (Fig. 6). ${ }^{18}$ F-fluorodeoxyglucose (FDG) positron emission tomography (PET)/CT of the whole body to detect the presence of non-CNS lymphoma showed abnormal FDG uptake in the right lateral ventricle of the brain with no evidence of systemic involvement (Fig. 7). The patient was treated with high-dose methotrexate (HD-MTX) for three cycles after the final diagnosis of PCNSL. The follow-up brain MRI showed complete resolution of the subependymal nodular enhancing lesions along the ventricles and hypothalamus (Fig. 8). However, the patient died from hospital-acquired, multidrug-resistant Klebsiella pneumoniae bacteremia 3 months after the PCNSL diagnosis, despite complete remission of PCNSL after HD-MTX treatment.

The study protocol was approved by the Institutional Review Board at Chonnam National University Hospital (CNUHEXP-2018-018).

\section{DISCUSSION}

The patient was diagnosed with IDD of the CNS before confirmation of DLBCL on brain biopsy. PCNSL rarely mimics IDD, including MS, NMOSD, and acute transverse myelitis (TM) [68]. Both conditions can present with acute neurological signs of localized contrast-enhancing lesions in the CNS, with no detectable cause in a routine blood or CSF analysis [9].

Furthermore, both conditions tend to improve after corticosteroid administration $[6,7]$. In this case, PCNSL was overlooked
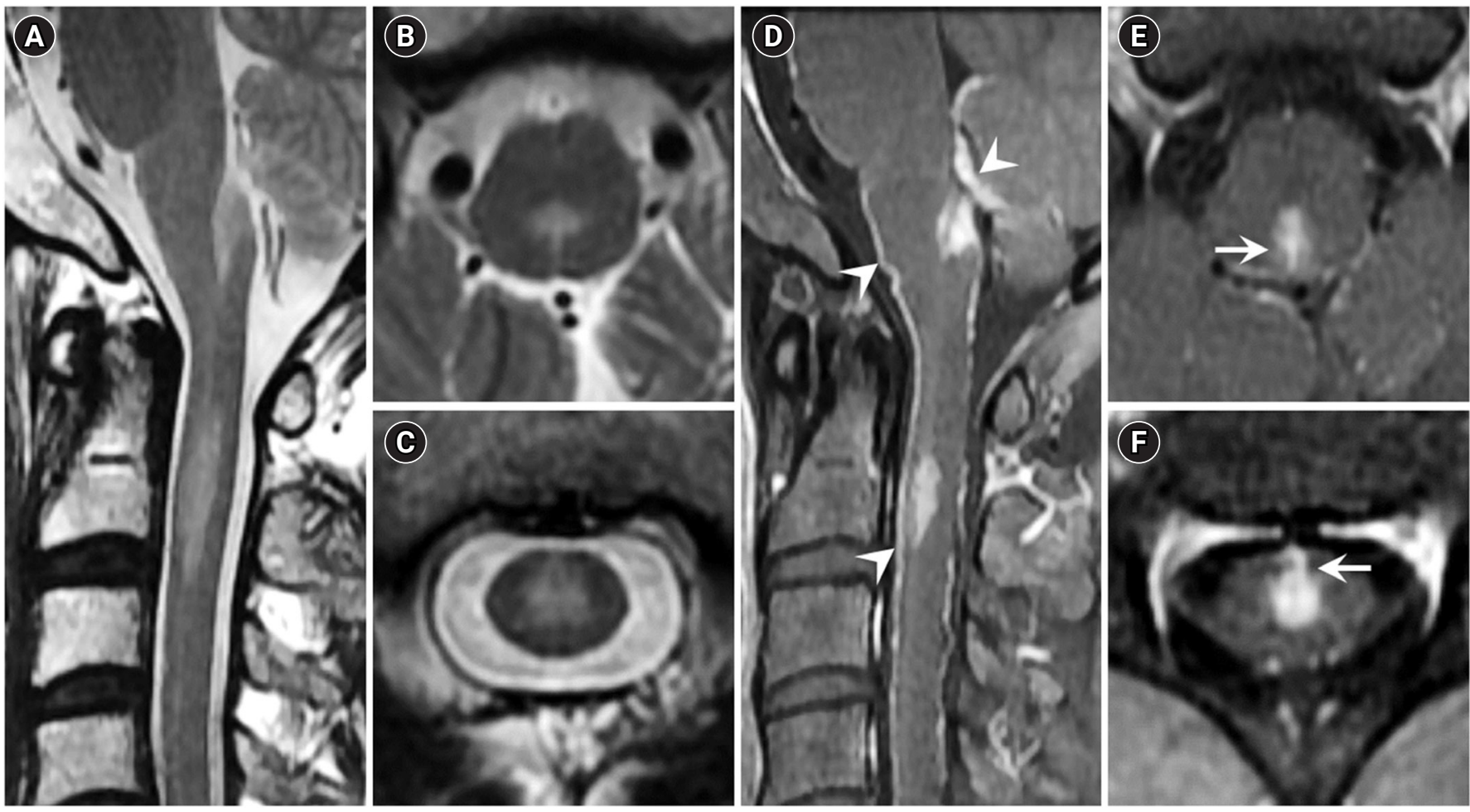

Fig. 2. Cervical spinal cord magnetic resonance imaging 3 weeks after the onset of symptoms. (A) Sagittal and (B, C) axial T2-weighted images show hyperintense lesions in the medulla oblongata and cervical spinal cord. (D) Gadolinium-enhanced T1-weighted images show leptomeningeal enhancement (arrowheads) and an intraparenchymal tadpole-like signal change (arrows) connected to the leptomeninges at the levels of (E) the medulla oblongata and (F) C2 vertebral body. 

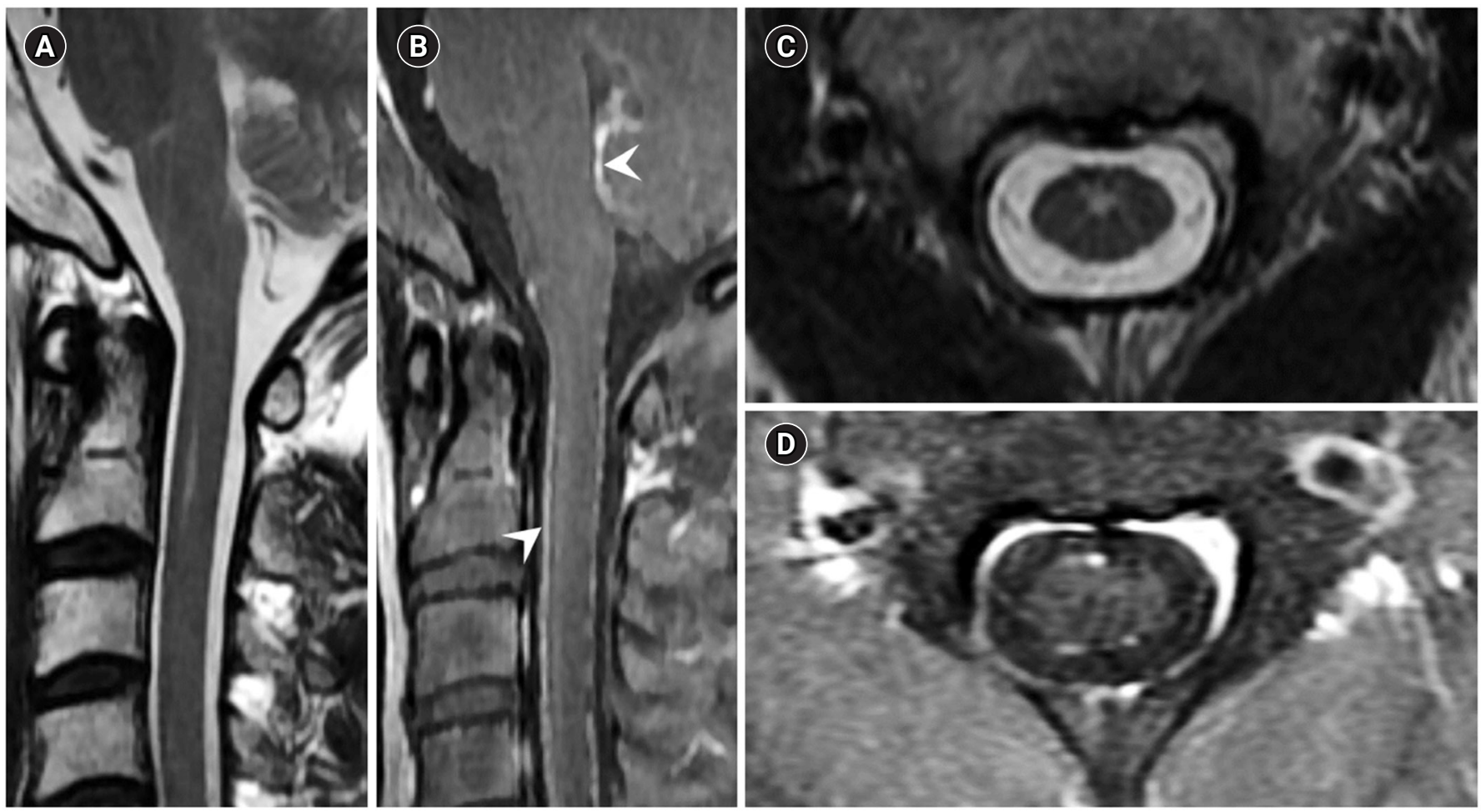

Fig. 3. Follow-up cervical spinal cord magnetic resonance imaging 6 months after the onset of symptoms. T2-weighted images $(A, C)$ show marked decrease in multifocal lesions with no parenchymal enhancement $(B, D)$. Gadolinium-enhanced T1-weighted sagittal images show persistent leptomeningeal enhancement ( $\mathrm{B}$, arrowheads).
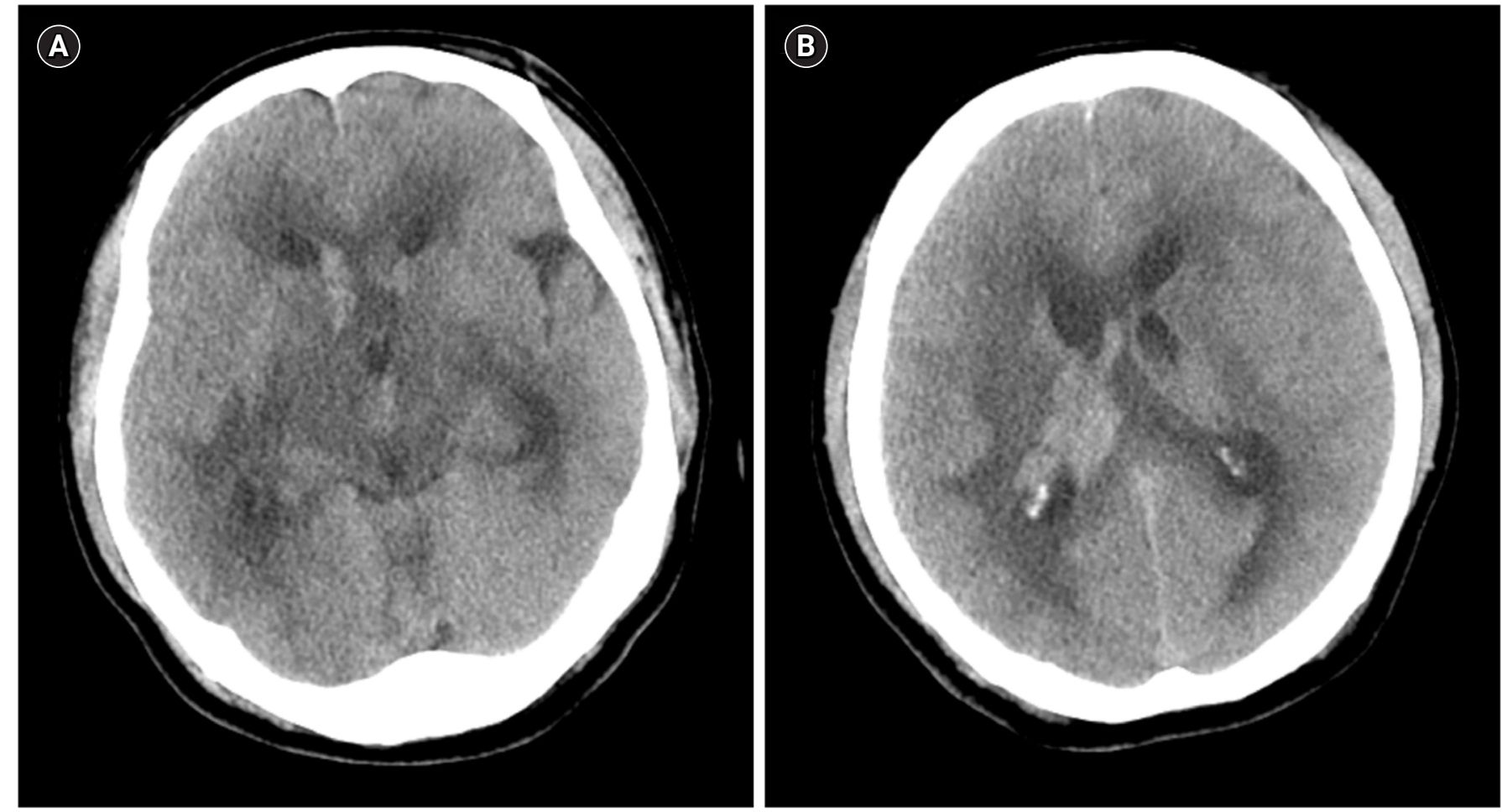

Fig. 4. Brain computed tomography (CT) at the onset of the semicomatose status. Brain CT shows subependymal hyperdense lesions in all ventricles, including the right lateral ventricle, with hydrocephalus (A), and edema in the periventricular white matter and cerebral peduncle (B). 

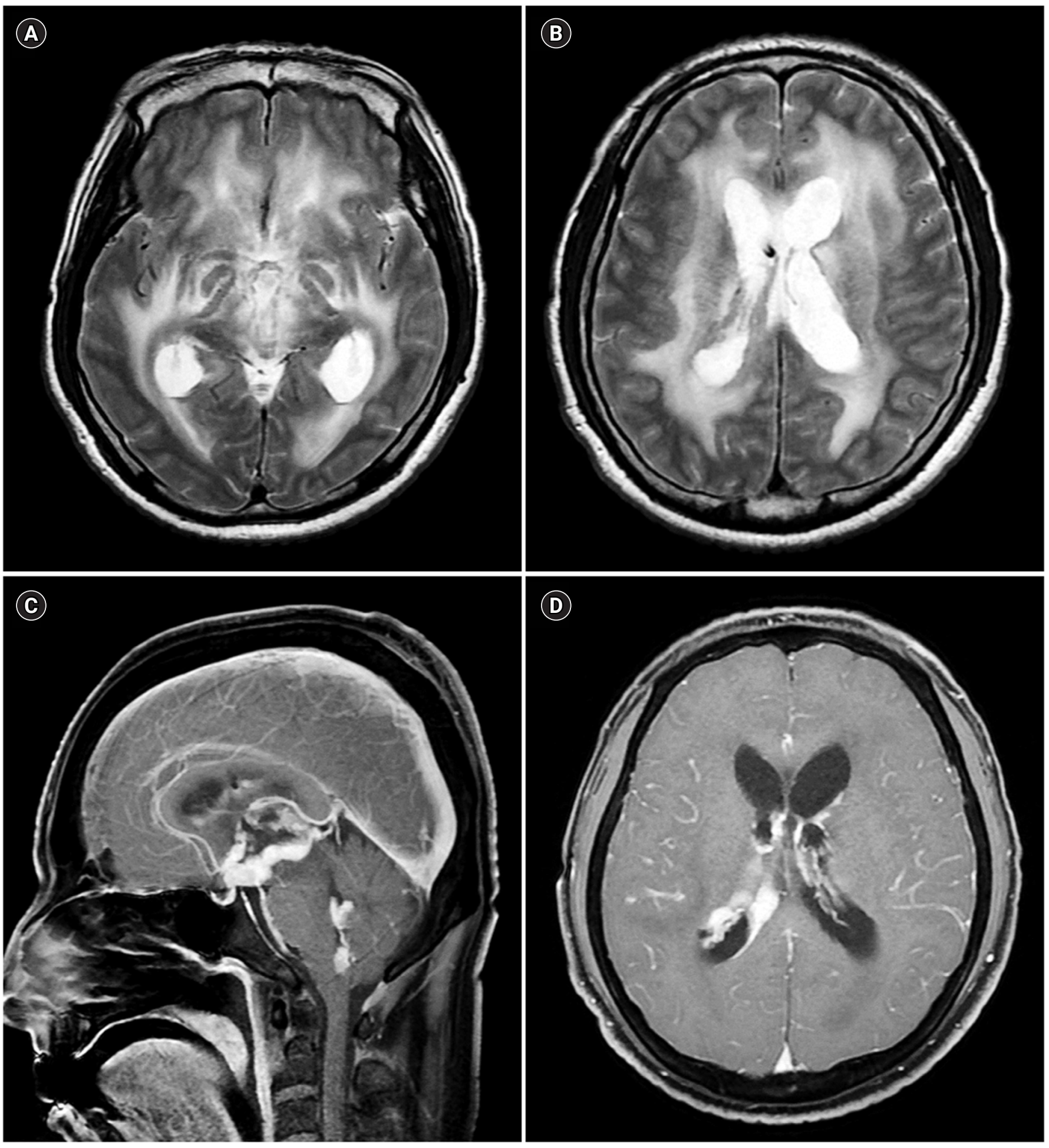

Fig. 5. Brain magnetic resonance imaging (MRI) after ventricular brain biopsy. (A, B) Axial T2-weighted images show hyperintensities in the hypothalamus and periventricular white matter. $(C, D)$ Gadolinium-enhanced MRI shows multifocal subependymal nodular enhancing lesions. 

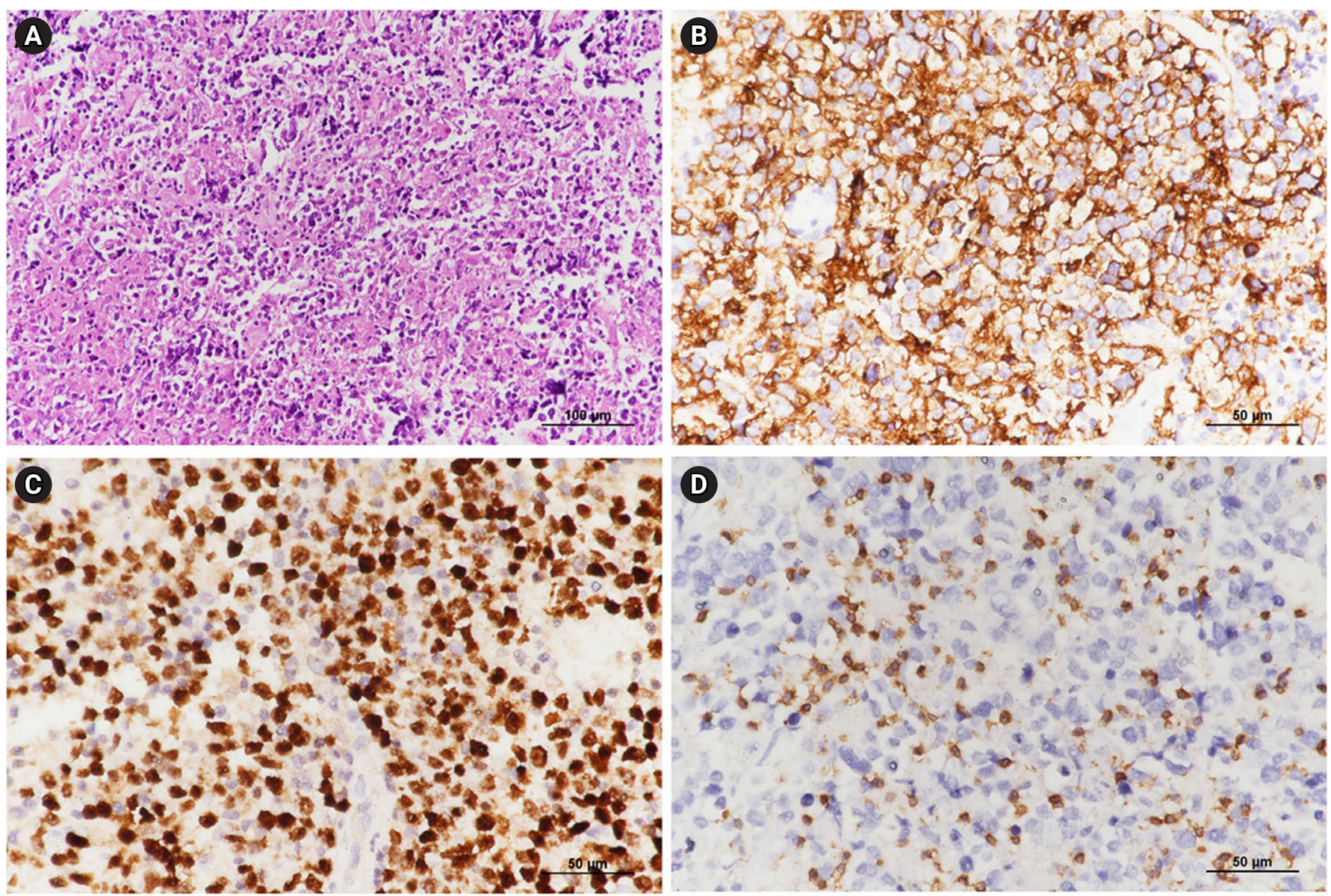

Fig. 6. Pathologic findings. Hematoxylin and eosin staining (A) reveals large atypical lymphocytes mixed with small mature lymphocytes. Tumor cells were positive for CD20 (B, B-cell marker), with the Ki-67 labeling index approaching 80\% (C), but negative for CD3 (D, T-cell marker) on immunohistochemical analysis (original magnification, $\times 200$ ).

for 8 months after the onset of symptoms for the following reasons: (1) immunocompetence and young age of the patient; (2) focal neurologic symptoms and signs suggesting acute TM; (3) unremarkable serial CSF cytomorphologic tests, with no exposure to corticosteroids; (4) improvement in symptoms and MRI lesions after corticosteroid treatment; and (5) initial CNS lesions confined to the intramedullary cervical spinal cord and vicinity of the medulla oblongata, which is rare in PCNSL [4-6]. Therefore, therapy with HD-MTX for PCNSL was delayed in this case.

The survival time and clinical outcome in patients with PCNSL have significantly improved since the introduction of HD-MTX, which can penetrate the blood-brain barrier, as the first-line chemotherapy regimen $[1,2,5]$. In this case, PCNSL was only suspected after deterioration in mental status because of tumor infiltration in the supratentorial white matter and ventricles. Therefore, HD-MTX chemotherapy was administrated 8 months after the onset of initial neurologic symptoms, and de- layed treatment for PCNSL may have caused the poor outcome. Treatment initiation in the early stages of PCNSL can improve the survival rate and clinical outcome before PCNSL spreads widely or infiltrates diffusely $[1,2]$, while PCNSL in the late stages is often refractory to HD-MTX chemotherapy, or can recur or progress to systemic involvement $[1,2,10]$. Therefore, early brain biopsy should be performed to diagnose DLBCL pathologically in patients suspected with PCNSL $[1,10]$, considering that precise diagnosis and early initiation of treatment are important for good prognosis in PCNSL. The patient's prognosis would have been better with HD-MTX chemotherapy administration before PCNSL spread or infiltrated other CNS regions. HD-MTX chemotherapy was effective in our case, as observed on follow-up MRI. However, early brain biopsy is not suitable for PCNSL-suspected patients with lesions in the intramedullary spinal cord because of risk of permanent postoperative neurological deficit [5]. Whole-body ${ }^{18}$ F-FDG-PET/CT can be performed to detect sys- 
A

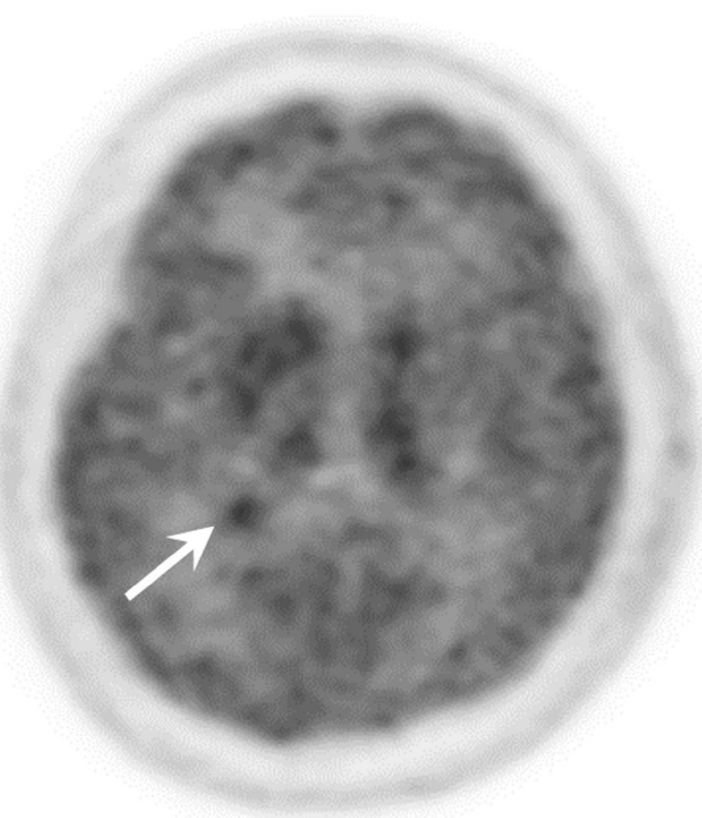

C

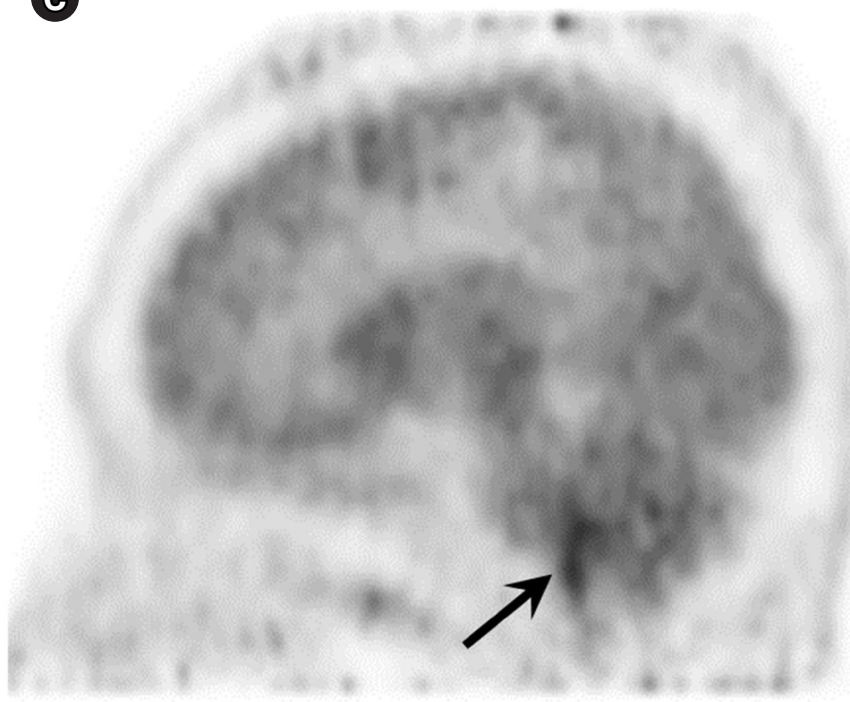

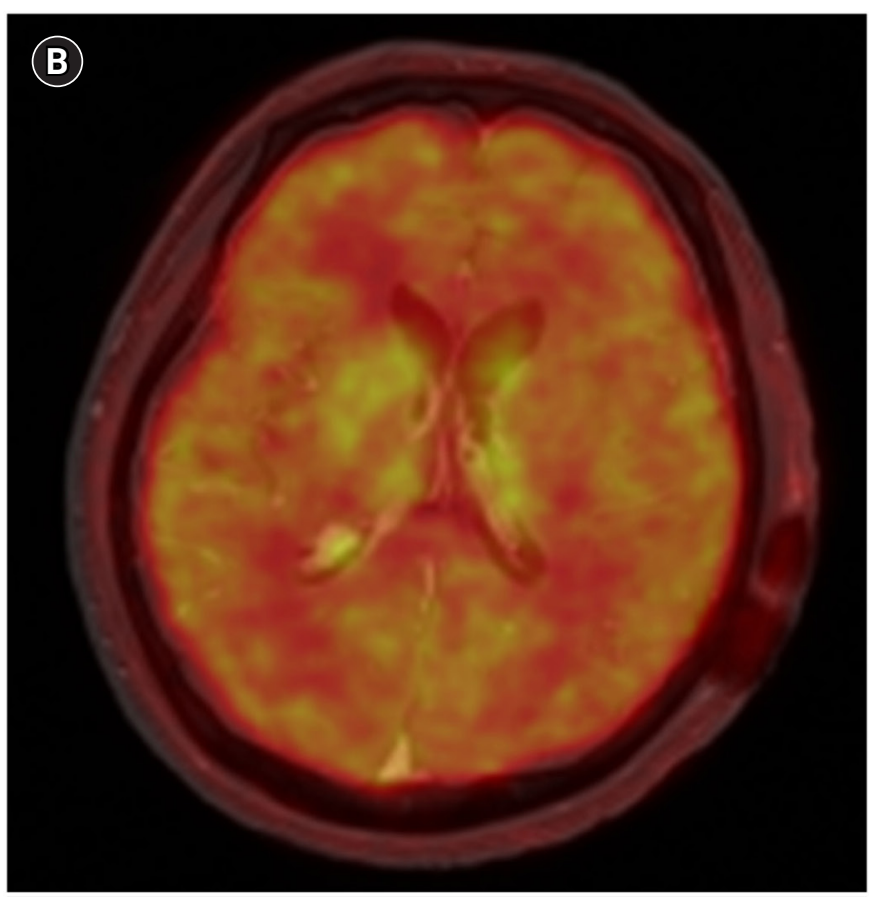
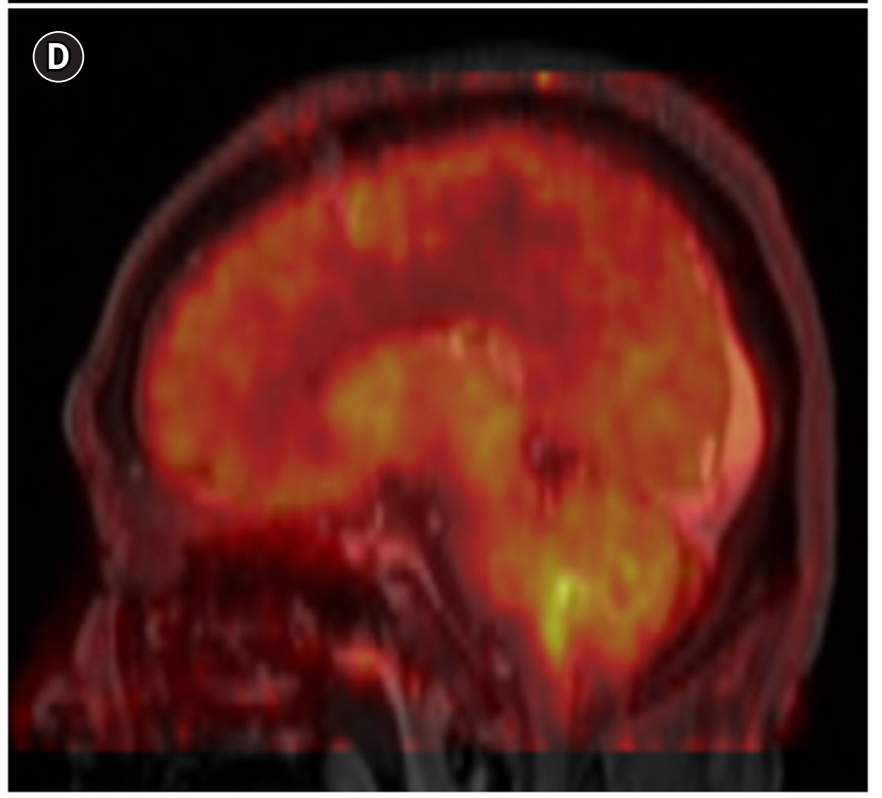

Fig. 7. ${ }^{18} \mathrm{~F}$-fluorodeoxyglucose (FDG)-positron emission tomography (PET) of the brain. ${ }^{18} \mathrm{~F}$-FDG-PET shows abnormal FDG uptake in the right lateral ventricle $(A$, white arrow) and inferior 4th ventricle ( $C$, black arrow). PET and coregistered PET/magnetic resonance fusion imaging shows asymmetric, mild hypermetabolic lesions along the body, atrium, and posterior horn of the right lateral ventricle with a maximum standardized uptake volume (SUVmax) of $4.4(\mathrm{~A}, \mathrm{~B})$, and moderate hypermetabolic lesions along the inferior 4th ventricle with an SUVmax of 5.6 (C, D).

temic lymphoma before corticosteroid administration in a PCNSL-suspected patient who has difficulty undergoing brain biopsy, as systemic involvement of DLBCL is found in 7\% of PCNSL-suspected patients at initial diagnosis [11]. However, studies on the usefulness of ${ }^{18} \mathrm{~F}$-FDG-PET/CT in differentiation of PCNSL from IDD is lacking [12]. The key radiologic features that help distinguish PCNSL with spinal cord involvement from IDD are: (1) multifocal intramedullary lesions with brain lesions [5]; (2) involvement of the conus medullaris or cauda equine [5]; (3) persistent Gd-enhancing intramedullary lesions for over 8 weeks [5]; (4) persistent, contiguous Gd-enhancing MRI lesions along the ventricular regions and spinal leptomeninges [4]; 

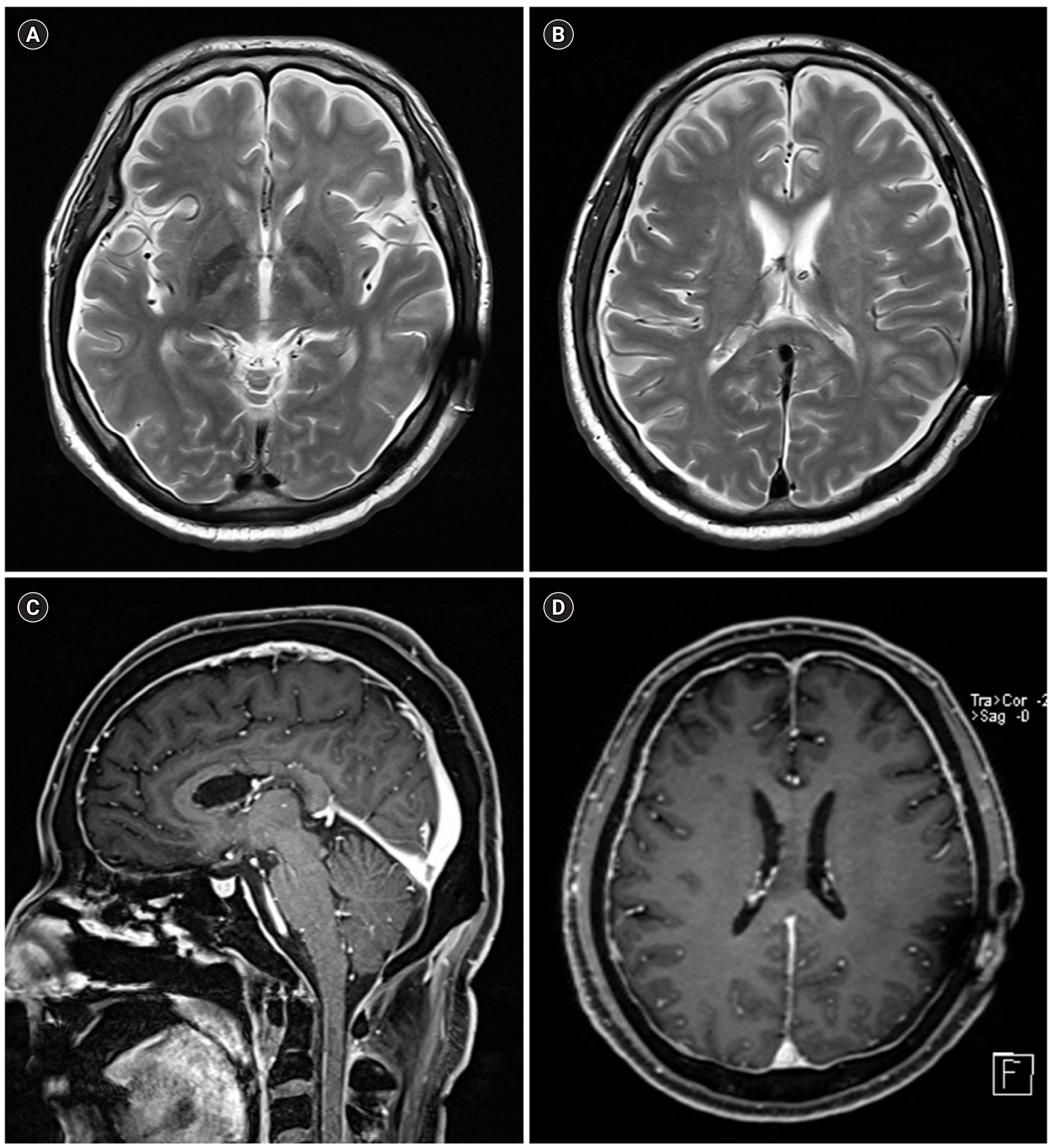

Fig. 8. Brain magnetic resonance imaging (MRI) after high-dose methotrexate chemotherapy. (A, B) Axial T2-weighted images show complete resolution of the multifocal subependymal nodular lesions in the hypothalamus and ventricular regions. (C, D) Gadoliniumenhanced MRI shows diffuse pachymeningeal thickening with no leptomeningeal enhancement. 
and (5) intraparenchymal tadpole-like enhancing lesions, which may be helpful in suspected PCNSL cases. In addition, CSF flow cytometry is superior to CSF cytomorphologic testing in PCNSL diagnosis $[13,14]$.

This case highlights that PCNSL with involvement of the intramedullary spinal cord can be misdiagnosed as IDD. The characteristic radiologic features, including persistent Gd-enhancing MRI lesions along the ventricular regions and spinal leptomeninges, may be helpful in differentiating PCNSL from IDD in early stages of the disease.

\section{ARTICLE INFORMATION}

\section{Conflict of interest}

No potential conflict of interest relevant to this article.

\section{Funding}

This work was supported by a grant (BCRI 19049) from Chonnam National University Hospital Biomedical Research Institute.

\section{ORCID}

Hyunsoo Kim, https://orcid.org/0000-0001-9340-8619

Tai-Seung Nam, https://orcid.org/0000-0003-2771-8728

Michael Levy, https://orcid.org/0000-0002-7969-8346

\section{Author contribution}

Conceptualization: TSN and ML. Data curation \& Formal analysis: HK, TSN, KHL, JK, and SJL. Visualization \& Writing-original draft: HK and TSN. Writing-review editing: TSN and ML.

\section{REFERENCES}

1. Grommes C, DeAngelis LM. Primary CNS lymphoma. J Clin Oncol 2017;35:2410-8.

2. Hochberg FH, Baehring JM, Hochberg EP. Primary CNS lymphoma. Nat Clin Pract Neurol 2007;3:24-35.

3. Sierra del Rio M, Rousseau A, Soussain C, Ricard D, Hoang-Xu- an K. Primary CNS lymphoma in immunocompetent patients. Oncologist 2009; 14:526-39.

4. Herrlinger U, Weller M, Küker W. Primary CNS lymphoma in the spinal cord: clinical manifestations may precede MRI detectability. Neuroradiology 2002;44:239-44.

5. Flanagan EP, O’Neill BP, Porter AB, Lanzino G, Haberman TM, Keegan BM. Primary intramedullary spinal cord lymphoma. Neurology 2011;77:784-91.

6. Elavarasi A, Dash D, Warrier AR, Bhatia R, Kumar L, Jain D, et al. Spinal cord involvement in primary CNS lymphoma. J Clin Neurosci 2018;47:145-8.

7. DeAngelis LM. Primary central nervous system lymphoma imitates multiple sclerosis. J Neurooncol 1990;9:177-81.

8. Kim SM, Kim SJ, Lee HJ, Kuroda H, Palace J, Fujihara K. Differential diagnosis of neuromyelitis optica spectrum disorders. Ther Adv Neurol Disord 2017;10:265-89.

9. Scott BJ, Douglas VC, Tihan T, Rubenstein JL, Josephson SA. A systematic approach to the diagnosis of suspected central nervous system lymphoma. JAMA Neurol 2013;70:311-9.

10. Han $\mathrm{CH}$, Batchelor TT. Primary central nervous system lymphoma. Continuum (Minneap Minn) 2017;23(6, Neuro-oncology):1601-18

11. Mohile NA, Deangelis LM, Abrey LE. The utility of body FDG PET in staging primary central nervous system lymphoma. Neuro Oncol 2008;10:223-8.

12. Chiavazza C, Pellerino A, Ferrio F, Cistaro A, Soffietti R, Rudà R. Primary CNS lymphomas: challenges in diagnosis and monitoring. Biomed Res Int 2018;2018:3606970.

13. Bromberg JE, Breems DA, Kraan J, Bikker G, van der Holt B, Smitt PS, et al. CSF flow cytometry greatly improves diagnostic accuracy in CNS hematologic malignancies. Neurology 2007;68:1674-9.

14. Cordone I, Masi S, Carosi M, Vidiri A, Marchesi F, Marino M, et al. Brain stereotactic biopsy flow cytometry for central nervous system lymphoma characterization: advantages and pitfalls. J Exp Clin Cancer Res 2016;35:128. 